\title{
Isotropic and squeezed colored pump noise effects on the degenerate parametric oscillator
}

\author{
L. Gilles, ${ }^{1}$ P. Tombesi, ${ }^{1}$ M. San Miguel, ${ }^{2}$ and P. García-Fernández ${ }^{3}$ \\ ${ }^{1}$ Dipartimento di Matematica e Fisica, Università di Camerino, I-62032 Camerino, Italy \\ ${ }^{2}$ Departament de Fí sica, Universitat de les Illes Balears, E-07071 Palma de Mallorca, Spain \\ ${ }^{3}$ Instituto de Estructura de la Materia, Consejo Superior de Investigaciones Cientificas, Serrano 123, 28006 Madrid, Spain
}

(Received 26 February 1996)

\begin{abstract}
The degenerate parametric oscillator above threshold is studied with phenomenological stochastic colored (nonwhite) pump noise for arbitrary pump to subharmonic relaxation rate. The current experimental limits of large intracavity threshold photon numbers (very small quantum noise) are considered, allowing for a semiclassical treatment of the system dynamics. A comparison between the effects of isotropic and squeezed pump noise on the internal transient and steady-state fluctuations is presented by simulation of the nonlinear semiclassical stochastic Langevin equations in the Wigner quadrature representation. It is found that the transient squeezing for the system starting in the unstable steady state (the vacuum) is not degraded by stochastic pump noise. A damped oscillatory behavior of the noise levels (periodic exchange of fluctuations between the squeezed quadrature of the signal and the pump) is observed for large damping of the signal in the turn on of pump depletion. Finally, it is shown that the limited squeezing above threshold in the steady state (50\%) due to pump depletion can be enhanced if squeezed stochastic noise with sufficient significant spectral components (broadband squeezing) is fed to the pump. The above-threshold steady-state squeezing has been calculated analytically from the linearized stochastic equations and the effects of the time scale associated to the relaxation of the pump noise (the noise correlation time) compared to the dissipative time scale of the system and the pump to subharmonic loss ratio are presented. [S1050-2947(96)03609-8]
\end{abstract}

PACS number(s): 42.65.Ky, 42.50.Dv, 42.50.Lc, 42.65.Sf.

\section{INTRODUCTION}

The degenerate parametric oscillator has been in recent years the subject of great interest as a nonlinear dissipative system able to produce a large amount of squeezing [1], revealing classical or quantum-mechanical behavior, depending on the chosen regime of operation. Classical treatments of parametric oscillators were given by Bloembergen [2] and Armstrong et al. [3]. Quantum-mechanical treatments were presented early by Graham and Haken [4], Graham [5] using the Wigner representation, and more recently by Drummond, McNeil, and Walls [6] using the positive- $P$ representation [7].

The quantum-classical correspondence can be addressed on the ground of a description of the system's evolution in terms of a system "size" parameter [8]. A natural choice for the system size parameter is given by the parametric nonlinearity to cavity loss ratio (coupling constant scaled by the geometric mean decay rate of the modes) defining the characteristic undepleted intracavity pump photon number at threshold, $n_{\text {th }}^{-1} \equiv \eta^{2} /\left(\gamma_{a} \gamma_{b}\right)$, where $\gamma_{a}, \gamma_{b}$ are the signal and pump mode relaxation rates, and $\eta$ is the phenomenological nonlinear mode coupling. Semiclassical regimes are found for weak parametric nonlinearities (compared to the cavity losses) $\eta^{2} \ll\left(\gamma_{a} \gamma_{b}\right)$, i.e., large $n_{\text {th }}$, usually referred to in the literature as the small $g^{2}$ limit, the limit of small quantum noise $\left[g^{2} \equiv\left(2 n_{\mathrm{th}}\right)^{-1}\right]$. In such regimes, the internal interaction time scale $\eta^{-1}$ is much larger than the dissipative time scale and the effect of quantum noise is to perturb around classical solutions (diffusion approximation [5]). This mode of operation, characterized by very large photon numbers at threshold, corresponds to the majority of optical experiments
[1]. On the other hand, for $\eta^{2} \gg\left(\gamma_{a} \gamma_{b}\right), n_{\text {th }}$ is very small and single quanta processes are significant, even in the presence of high pump intensities (large intracavity photon numbers). For such regimes of strong coupling, reversible and irreversible dynamics cannot be separated anymore into a semiclassical evolution perturbed by small quantum fluctuations. This is the case for microwave Josephson oscillators that have high nonlinearities [9]. Such extreme nonlinear quantum devices characterized by a very small threshold photon number (large quantum noise) have been the subject of recent interest by the possibility they offer to produce quantum superposition states ("Schrödinger-cat" states) in dissipative environments [10]. Analytical solutions valid for arbitrary quantum noise strength have been obtained using the positive- $P$ representation, in the adiabatic limit where the pump mode decays much faster than the subharmonic, by Drummond et al. [11] and by Wolinsky and Carmichael [12].

In this paper, the process of subharmonic generation is studied, involving the nonlinear interaction of a quantized light field mode (the pump $\hat{b}$ ) at central frequency $2 \Omega$ with its subharmonic (the signal $\hat{a}$ ) at central frequency $\Omega$ in a nonlinear cristal described by a second-order susceptibility $\chi^{(2)}$ placed inside an optical cavity. The second-order susceptibility is the nonlinear response of the material to the application of two input optical fields [13]. An external classical pumping $\mathcal{E}_{b}$ is applied to drive the higher frequency mode $\hat{b}$ and bring the system to a nonequilibrium steady state. The coupling of the internal field modes to the external continuum of modes is modeled through the transmissivity of the cavity mirror, leading to the relaxation of the pump and the signal modes (relaxation rates $\gamma_{b}, \gamma_{a}$ ) [14]. Above threshold, the system displays bistability, the signal becomes 
excited and grows in amplitude with two possible phases separated by $\pi$, while the below-threshold solution (zero coherent amplitude) becomes unstable. The potential use of the bistable behavior of the parametric oscillator as an optical switch (phase switching) has attracted several investigations [15]. Even in the absence of thermal fluctuations, the system may tunnel from one amplitude to the other due to the presence of quantum noise as calculated by Kinsler and Drummond [16] in the small quantum noise limit.

The aim of this paper is to investigate the effects of the presence of phenomenological stochastic colored (nonwhite) fluctuations of the external classical pumping $\mathcal{E}_{b}$ on the squeezing produced above threshold. The cases of isotropic (phase unsensitive) and squeezed (phase sensitive) colored pump noise are considered. Apart from a quantum treatment of the nondegenerate parametric oscillator with squeezed vacuum input to the pump mode [17] using the generalized$P$ representation, no study of this problem seems to have been carried out. Attention is focused on the current experimental limits of large intracavity threshold optical photon numbers, $n_{\mathrm{th}} \sim 10^{10}$ (very small quantum noise), allowing for a semiclassical treatment of the system dynamics in the Wigner representation [18] (symmetrically ordered operators). This approximation is valid at optical frequencies but not for oscillators operating in the microwave region (microcavity configurations). Simulation of the nonlinear semiclassical stochastic Langevin equations in the Wigner quadrature representation (including spontaneous emission) with two statistically independent added colored Gaussian pump quadrature noise terms describing phenomenological stochastic fluctuations of the complex driving amplitude $\mathcal{E}_{b}$, and analytical steady-state results obtained from the linearized equations are presented. Gaussian white and colored (nonwhite), isotropic and squeezed (nonisotropic) stochasticity in the Langevin equations is considered. Squeezing in the pump could be achieved by mixing a coherent amplitude with a squeezed vacuum produced by a parametric amplifier operated below threshold in a single-ended cavity [14]. The effects of the time scales associated to the relaxation of the pump noise (the noise correlation time) compared to the dissipative time scale $\gamma_{a}^{-1}$ of the system and the pump to subharmonic loss ratio $r \equiv \gamma_{b} / \gamma_{a}$ are discussed within this framework.

The remainder of the paper is organized as follows. In Sec. II, the description of the quantum-mechanical model Hamiltonian and the semiclassical Langevin equations in the Wigner quadrature representation are given. The stochastic pump field is introduced from a semiclassical point of view. In Sec. III, steady states and their stability are revised. The effects of stochastic pump on the linearized internal quadrature variances are examined. In the case of squeezed stochastic pumping, the effects of the finite squeezing bandwidth $\gamma$ of the noise fed to the pump (compared to the signal mode bandwidth $\gamma_{a}$ ) are analyzed. Results for the transient fluctuations are presented from the numerical simulations of the nonlinear semiclassical equations in Sec. IV. A damped oscillatory behavior of the noise levels (periodic exchange of fluctuations between the squeezed quadrature of the signal and the pump) is observed for large damping of the signal in the turn on of pump depletion. It is found that the transient squeezing [19] for the system starting in the unstable steady state (the vacuum) is not degraded by stochastic pump noise. It is shown that the limited squeezing above threshold in the steady state (50\%) due to pump depletion [20] can be enhanced if squeezed stochastic noise is fed to the pump in an appropriate quadrature, as one should expect. Section V gives a summary and conclusions.

\section{THE MODEL SYSTEM}

The interaction of a light field mode $\hat{b}$ at frequency $2 \Omega$ (the pump mode) with another degenerate field mode $\hat{a}$ at frequency $\Omega$ (the subharmonic mode) in a nonlinear crystal placed inside a resonant optical ring cavity configuration with only one port, used for both the input and the output, is considered. The coupling of the internal harmonic and subharmonic cavity modes to the external environment leads to the decay rates $\gamma_{a}, \gamma_{b}$. The harmonic mode is pumped by a classical driving field. This process may be described by the following Hamiltonian in the Schrödinger picture $[\hbar=1]$ :

$$
\begin{gathered}
\hat{H}=\hat{H}_{S}+\hat{H}_{R}+\hat{H}_{S R}, \\
\hat{H}_{S}=\hat{H}_{0}+\hat{H}_{1}, \\
\hat{H}_{0}=\Omega \hat{a}^{\dagger} \hat{a}+2 \Omega \hat{b}^{\dagger} \hat{b}, \\
\hat{H}_{1}=-i \frac{\eta}{2}\left\{\hat{a}^{2} \hat{b}^{\dagger}-\text { H.c. }\right\}+i \mathcal{E}_{b}\left\{\hat{b}^{\dagger} \exp (-2 i \Omega t-i \theta)-\text { H.c. }\right\} \\
\hat{H}_{R}=\sum_{k}\left\{\omega_{a k} \hat{r}_{a k}^{\dagger} \hat{r}_{a k}+\omega_{b k} \hat{r}_{b k}^{\dagger} \hat{r}_{b k}\right\}, \\
\hat{H}_{S R}=\left(\hat{a} \hat{\Gamma}_{a}^{\dagger}+\text { H.c. }\right)+\left(\hat{b} \hat{\Gamma}_{b}^{\dagger}+\text { H.c. }\right),
\end{gathered}
$$

where $\hat{H}_{S}, \hat{H}_{R}$, and $\hat{H}_{S R}$ represent, respectively, the system Hamiltonian, i.e, the harmonic and subharmonic free fields plus the parametric intermode coupling and the driving of mode $\hat{b}$, the two free baths, and the system-baths coupling Hamiltonians. $\mathcal{E}_{b}$ is proportional to the modulus of the coherent part of the complex driving amplitude (whose phase is $\theta$ ) and represents the rate at which pump photons are injected into the fundamental (pump) mode. The nonlinear interaction removes single photons from the pump mode and creates pairs of photons in the subharmonic at a rate $\eta$ (chosen real and positive). $\hat{\Gamma}_{a}, \hat{\Gamma}_{b}$ are independent bath mode operators (quantum white noise operators),

$$
\hat{\Gamma}_{z}(t)=-i \sum_{k} \chi_{z, k} \hat{r}_{z, k}(0) \exp \left\{i\left(\Omega-\omega_{z, k}\right) t\right\}, \quad z=a, b
$$

of zero mean value and white-noise delta correlations

$$
\left\langle\hat{\Gamma}_{z}(t) \hat{\Gamma}_{z^{\prime}}^{\dagger}\left(t^{\prime}\right)\right\rangle=\gamma_{z} \delta_{z, z^{\prime}} \delta\left(t-t^{\prime}\right),
$$

describing the coupling of the cavity modes to the vacuum fluctuations entering the cavity by the partially transmitting mirror, leading to the loss of photons from the cavity at rates $\gamma_{a}, \gamma_{b}$ (fluctuation-dissipation theorem) [21] for the subharmonic and fundamental modes. 
The complex exponential in the parametric Hamiltonian (1) can be easily removed by working in the interaction picture defined by the transformation $\hat{b} \exp (2 i \Omega t+i \theta) \rightarrow \hat{b}$, $\hat{a} \exp (i \Omega t+i \theta / 2) \rightarrow \hat{a}$. In this rotating frame, following the standard theory of damping, nonlinear equations of motion for the system and bath operators are derived. After elimination of the bath modes using the Wigner-Weisskopff approximation, the resulting nonlinear quantum Langevin equation for the system canonical operators can be expressed as [22]

$$
\begin{gathered}
\dot{\hat{a}}=\eta \hat{a}^{\dagger} \hat{b}-\gamma_{a} \hat{a}+\hat{\Gamma}_{a}, \\
\dot{\hat{b}}=-\eta \hat{a}^{2} / 2+\mathcal{E}_{b}-\gamma_{b} \hat{b}+\hat{\Gamma}_{b} .
\end{gathered}
$$

The Langevin equations (4) are written in scaled variables as

$$
\begin{gathered}
d \hat{a} / d \tau=-\hat{a}+\tilde{\eta} \hat{a}^{\dagger} \hat{b}+\hat{\xi}_{a}(\tau), \\
d \hat{b} / d \tau=-r \hat{b}-\tilde{\eta} \hat{a}^{2} / 2+\widetilde{\mathcal{E}}_{b}+\hat{\xi}_{b}(\tau),
\end{gathered}
$$

where we have defined

$$
\begin{gathered}
\tau=\gamma_{a} t, \\
r=\frac{\gamma_{b}}{\gamma_{a}}, \\
\widetilde{\eta}=\eta / \gamma_{a}, \\
\widetilde{\mathcal{E}}_{b}=\mathcal{E}_{b} / \gamma_{a}, \\
\hat{\xi}_{a}(\tau)=\hat{\Gamma}_{a}(t) / \gamma_{a}, \\
\hat{\xi}_{b}(\tau)=\hat{\Gamma}_{b}(t) / \gamma_{a} .
\end{gathered}
$$

Making use of the system size expansion [8] (regime of weak coupling) we can transform these nonlinear quantum Langevin equations into complex nonlinear stochastic equations for the process $(a, b)$ expressed in the Wigner representation (associated with symmetric ordering of operators):

$$
\begin{gathered}
d a / d \tau=-a+\tilde{\eta} a^{*} b+\xi_{a}, \\
d b / d \tau=-r b-\tilde{\eta} a^{2} / 2+\widetilde{\mathcal{E}}_{b}+\xi_{b} .
\end{gathered}
$$

The derivation of Eqs. (7) from Eqs. (5) relies on the truncation of third-order derivative terms in the corresponding Wigner Fokker-Planck equation. This approximation [5,23] is justified in the present case of very large $n_{\text {th }}$ (small $g^{2}$ limit).

Changing from the complex process $(a, b)$ to the fourcomponent real process $\left(x_{a}, y_{a}, x_{b}, y_{b}\right)$ defined by

$$
z=x_{z}+i y_{z}, \quad z=a, b,
$$

where $x_{z}$ and $y_{z}$ represent the two quadratures of the mode, and writing $\xi_{z}=\xi_{x_{z}}+i \xi_{y_{z}}$, we obtain the following nonlinear stochastic equations:

$$
\begin{gathered}
\dot{x}_{a}=-x_{a}+\widetilde{\eta}\left[x_{a} x_{b}+y_{a} y_{b}\right]+\xi_{x_{a}}(\tau), \\
\dot{y}_{a}=-x_{a}+\widetilde{\eta}\left[x_{a} y_{b}-y_{a} x_{b}\right]+\xi_{y_{a}}(\tau), \\
\dot{x}_{b}=-r x_{b}-\widetilde{\eta}\left[x_{a}^{2}-y_{a}^{2}\right] / 2+\widetilde{\mathcal{E}}_{b}+\xi_{x_{b}}(\tau), \\
\dot{y}_{b}=-r y_{b}-\widetilde{\eta} x_{a} y_{a}+\xi_{y_{b}}(\tau),
\end{gathered}
$$

where we have defined independent real white noises $\xi_{u_{a}}(\tau), \xi_{u_{b}}(\tau), u=x, y$ describing spontaneous emission with correlations

$$
\begin{aligned}
\left\langle\xi_{u_{z}}(\tau) \xi_{u_{z}}\left(\tau^{\prime}\right)\right\rangle & =\left\langle\hat{\xi}_{u_{z}}(\tau) \hat{\xi}_{u_{z}}\left(\tau^{\prime}\right)\right\rangle_{\mathrm{sym}} \\
& =\frac{1}{2} \delta\left(\tau-\tau^{\prime}\right) \quad \text { if } z=a \\
& =\frac{r}{2} \delta\left(\tau-\tau^{\prime}\right) \quad \text { if } z=b
\end{aligned}
$$

The contribution of the coherent part of the pump is assumed to be also included in the white noise sources $\xi_{u_{b}}$. In order to describe the effects of a phenomenological additional incoherent stochastic part of the classical pump, the last equation in (7) is supplemented with a complex Gaussian colored noise term $\varepsilon_{b}=\varepsilon_{x_{b}}+i \varepsilon_{y_{b}}$, of zero mean value, i.e., the replacement $\widetilde{\mathcal{E}_{b}} \rightarrow \widetilde{\mathcal{E}}_{b}+\varepsilon_{b}(\tau)$ is made, transforming (9) into

$$
\begin{gathered}
\dot{x}_{a}=-x_{a}+\widetilde{\eta}\left[x_{a} x_{b}+y_{a} y_{b}\right]+\xi_{x_{a}}(\tau), \\
\dot{y}_{a}=-x_{a}+\widetilde{\eta}\left[x_{a} y_{b}-y_{a} x_{b}\right]+\xi_{y_{a}}(\tau), \\
\dot{x}_{b}=-r x_{b}-\widetilde{\eta}\left[x_{a}^{2}-y_{a}^{2}\right] / 2+\widetilde{\mathcal{E}}_{b}+\varepsilon_{x_{b}}(\tau)+\xi_{x_{b}}(\tau), \\
\dot{y}_{b}=-r y_{b}-\widetilde{\eta} x_{a} y_{a}+\varepsilon_{y_{b}}(\tau)+\xi_{y_{b}}(\tau) .
\end{gathered}
$$

A comparison between the effects of isotropic and squeezed stochastic noise fed to the pump is considered. The source of squeezing is taken to be a degenerate parametric amplifier (DPA) operating below threshold in a single-ended cavity of damping $\gamma$ and resonant frequency $2 \Omega$. In a frame rotating at the carrier frequency $2 \Omega$, the squeezed pump noise contribution is described by the correlation functions [14]

$$
\begin{aligned}
\left\langle\varepsilon_{x_{b}}(\tau) \varepsilon_{x_{b}}\left(\tau^{\prime}\right)\right\rangle= & r\left\{-\sin ^{2}(\varphi) \frac{d_{+}}{\tau_{+}} e^{-\left|\tau-\tau^{\prime}\right| \tau_{+}^{-1}}\right. \\
& \left.+\cos ^{2}(\varphi) \frac{d_{-}}{\tau_{-}} e^{-\left|\tau-\tau^{\prime}\right| \tau_{-}^{-1}}\right\}, \\
\left\langle\varepsilon_{y_{b}}(\tau) \varepsilon_{y_{b}}\left(\tau^{\prime}\right)\right\rangle= & r\left\{-\cos ^{2}(\varphi) \frac{d_{+}}{\tau_{+}} e^{-\left|\tau-\tau^{\prime}\right| \tau_{+}^{-1}}\right. \\
& \left.+\sin ^{2}(\varphi) \frac{d_{-}}{\tau_{-}} e^{-\left|\tau-\tau^{\prime}\right| \tau_{-}^{-1}}\right\},
\end{aligned}
$$

where $\varphi$ controls the relative direction of squeezing (the phase of the squeezed vacuum relative to the phase of the oscillator coherent driving field). The intensities and normalized correlation times of the noise sources are given by 


$$
d_{ \pm}=\mu /\left(4 \lambda_{ \pm}^{2}\right), \quad \tau_{ \pm}=\xi \lambda_{ \pm}^{-1}
$$

with $\lambda_{ \pm}=(1 \pm \mu) / 2$ and $\xi=\gamma_{a} / \gamma$. The scaled pump parameter $\mu(0 \leqslant \mu<1)$ defines the strength of the squeezed pump noise (the external amplifier driving strength). Strong squeezing is achieved in one quadrature of the pump (the $y$ quadrature if $\varphi=0$ ), over a finite bandwidth proportional to $\xi^{-1}$, as threshold is approached, i.e., as $\mu \rightarrow 1$. In this limit, fluctuations in the unsqueezed quadrature become very large, and their correlation time $\tau_{-}$approaches infinity. The isotropic stochastic pump noise contribution will be described by the correlation functions

$$
\left\langle\varepsilon_{u_{b}}(\tau) \varepsilon_{u_{b}}\left(\tau^{\prime}\right)\right\rangle=\frac{r d}{\tau_{c}} e^{-\left|\tau-\tau^{\prime}\right| \tau_{c}^{-1}}, \quad u=x, y,
$$

and the cases $d=d_{ \pm}, \tau=\tau_{ \pm}$will be discussed. The pump to subharmonic loss ratio $r$ is present in (12) and (14) in order to fulfill the fluctuation-dissipation theorem because the above correlations are considered inside the cavity.

\section{LINEARIZED STEADY-STATE ANALYSIS}

\section{A. Deterministic steady-state solutions and stability analysis}

The deterministic steady-state mean value equations follow directly from (7) (without the noise terms) with the time derivatives of $a$ and $b$ set to zero, leading to

$$
\begin{gathered}
a_{s}=\widetilde{\eta} a_{s}^{*} b_{s}, \\
r b_{s}=-\tilde{\eta} a_{s}^{2} / 2+\widetilde{\mathcal{E}}_{b} .
\end{gathered}
$$

From Eqs. (15), the following steady-state solutions arise: (i) the trivial solution $a_{s}=0, b_{s}=\widetilde{\mathcal{E}}_{b} / r$; (ii) $a_{s} \neq 0$. Writing $a_{s}=\left|a_{s}\right| e^{i \phi}$, Eqs. (15) yield

$$
\begin{gathered}
\left(\widetilde{\eta}^{2}\left|a_{s}\right|^{2}\right) /(2 r)=\lambda-1 \quad(\lambda>1), \\
\phi=0, \quad 0+\pi \\
\widetilde{\eta} b_{s}=1 .
\end{gathered}
$$

The scaled pump parameter $\lambda \equiv\left(\widetilde{\eta} \widetilde{\mathcal{E}}_{b}\right) / r$ characterizes the pumping level, $\lambda=1$ corresponding to the critical pump power required to push the system into oscillation (the threshold for parametric oscillations). Above threshold $(\lambda>1)$, the semiclassical solutions (16) exhibit bistability. Two amplitudes with a phase separation of $\pi$ are possible.

To establish the stability and fluctuations of the steadystate solutions, the stochastic Langevin equations (7) are linearized around the steady states. This approximation remains valid as long as the fluctuations are much smaller than the classical mean values, which is the essence of the small $g^{2}$ limit (very small quantum noise). Writing the semiclassical mode amplitudes $a, b$ as the sum of the steady-state amplitude and a small perturbation around the steady states, $a(\tau)=a_{s}+\delta a(\tau), b(\tau)=b_{s}+\delta b(\tau)$, and substituting these expressions into (7), the following set of linearized equations for the small perturbations (neglecting second- and higherorder terms in $\delta a$ and $\delta b)$ is readily obtained:

$$
\begin{gathered}
d(\delta a) / d \tau=-\delta a+\widetilde{\eta}\left(a_{s}^{*} \delta b+b_{s} \delta a^{*}\right)+\xi_{a}, \\
d(\delta b) / d \tau=-r \delta b-\tilde{\eta} a_{s} \delta a+\varepsilon_{b}+\xi_{b} .
\end{gathered}
$$

The linearization procedure decouples the quadrature components of the field and leads to

$$
\begin{gathered}
\frac{d}{d \tau} \delta \mathbf{x}(\tau)=\mathcal{A}_{x} \delta \mathbf{x}+\mathcal{L}_{x}, \\
\frac{d}{d \tau} \delta \mathbf{y}(\tau)=\mathcal{A}_{y} \delta \mathbf{y}+\mathcal{L}_{y},
\end{gathered}
$$

where $\mathbf{x}=\left[x_{a}, x_{b}\right]^{T}, \mathbf{y}=\left[y_{a}, y_{b}\right]^{T}$, and the drift matrices are given by

$$
\mathcal{A}_{x}=\left[\begin{array}{cc}
\tilde{\eta} b_{s}-1 & \tilde{\eta} a_{s} \\
-\tilde{\eta} a_{s} & -r
\end{array}\right], \quad \mathcal{A}_{y}=\left[\begin{array}{cc}
-\tilde{\eta} b_{s}-1 & \tilde{\eta} a_{s} \\
-\tilde{\eta} a_{s} & -r
\end{array}\right] .
$$

The real noises $\mathcal{L}_{u}, u=x, y$ are written as a sum of independent white and colored noise sources,

$$
\begin{gathered}
\mathcal{L}_{u}=\mathcal{L}_{u}^{w}+\mathcal{L}_{u}^{c}, \\
\mathcal{L}_{u}^{w}=\left[\xi_{u_{a}}, \xi_{u_{b}}\right]^{T}, \\
\mathcal{L}_{u}^{c}=\left[0, \varepsilon_{u_{b}}\right]^{T},
\end{gathered}
$$

with the correlation properties

$$
\begin{gathered}
\left\langle\mathcal{L}_{u}^{w}(\tau) \mathcal{L}_{u}^{w^{T}}\left(\tau^{\prime}\right)\right\rangle=\mathcal{D}^{w} \delta\left(\tau-\tau^{\prime}\right), \\
\mathcal{D}^{w}=\frac{1}{2} \operatorname{diag}[1, r], \\
\left\langle\mathcal{L}_{u}^{c}(\tau) \mathcal{L}_{u}^{c^{T}}\left(\tau^{\prime}\right)\right\rangle=\mathcal{D}_{u}^{c}\left(\left|\tau-\tau^{\prime}\right|\right), \\
\mathcal{D}_{u}^{c}\left(\left|\tau-\tau^{\prime}\right|\right)=\operatorname{diag}\left[0,\left\langle\varepsilon_{u_{c}}(\tau) \varepsilon_{u_{c}}\left(\tau^{\prime}\right)\right\rangle\right],
\end{gathered}
$$

where the colored noise diffusion matrix $\mathcal{D}_{u}^{c}$ is given by (12) or (14) depending on whether squeezed or isotropic colored pump noise is considered.

The stability of the steady states is determined by investigating the eigenvalues of the drift matrices $\mathcal{A}_{x}, \mathcal{A}_{y}$. For the solution (i), the drift matrices are diagonal,

$$
\mathcal{A}_{x}=\operatorname{diag}[\lambda-1,-r], \quad \mathcal{A}_{y}=\operatorname{diag}[-\lambda-1,-r],
$$

and the eigenvalues are all negative provided $\lambda<1$. For the solution (ii), the drift matrices read

$$
\begin{aligned}
& \mathcal{A}_{x}=\left[\begin{array}{cc}
0 & \sqrt{2 r(\lambda-1)} \\
-\sqrt{2 r(\lambda-1)} & -r
\end{array}\right], \\
& \mathcal{A}_{y}=\left[\begin{array}{cc}
-2 & \sqrt{2 r(\lambda-1)} \\
-\sqrt{2 r(\lambda-1)} & -r
\end{array}\right],
\end{aligned}
$$

and their eigenvalues are 


$$
\begin{gathered}
\theta_{ \pm}^{x}=-\frac{r}{2} \pm \frac{1}{2} \sqrt{r^{2}-8 r(\lambda-1)}, \\
\theta_{ \pm}^{y}=-\frac{(2+r)}{2} \pm \frac{1}{2} \sqrt{(2+r)^{2}-8 r \lambda .} .
\end{gathered}
$$

These eigenvalues may present a complex character with a finite negative real part, ensuring the stability of the solution (ii). At the point $\lambda=1$, the eigenvalue $\theta_{+}^{x}$ becomes zero, and the system undergoes a pitchfork bifurcation. The solution (i) becomes unstable, and the system moves onto one of the two new stable branches given by the solution (ii).

\section{B. Fluctuations around the steady states}

The fluctuations around the steady states are obtained by integration of the stochastic equations (18):

$$
\begin{aligned}
& \delta \mathbf{x}(\tau)=e^{\mathcal{A}_{x} \tau} \delta \mathbf{x}(0)+\int_{0}^{\tau} e^{\mathcal{A}_{x}\left(\tau-\tau^{\prime}\right)} \mathcal{L}_{x}\left(\tau^{\prime}\right) d \tau^{\prime}, \\
& \delta \mathbf{y}(\tau)=e^{\mathcal{A}_{y} \tau} \delta \mathbf{y}(0)+\int_{0}^{\tau} e^{\mathcal{A}_{y}\left(\tau-\tau^{\prime}\right)} \mathcal{L}_{y}\left(\tau^{\prime}\right) d \tau^{\prime}
\end{aligned}
$$

The white noise case $\mathcal{L}_{u}=\mathcal{L}_{u}^{w}$ (coherent pumping) will be considered first. Using standard results for linear multivariate Ornstein-Uhlenbeck processes [24], the stationary correlation matrix $\Sigma_{u} \equiv \lim _{\tau \rightarrow \infty}\left\langle\delta \mathbf{u}(\tau) \delta \mathbf{u}^{T}(\tau)\right\rangle, u=x, y$ is obtained by solving the matrix equation

$$
\mathcal{A}_{u} \Sigma_{u}^{w}+\Sigma_{u}^{w} \mathcal{A}_{u}^{T}=-\mathcal{D}^{w},
$$

where $\Sigma_{u}^{w}$ denotes the correlation matrix averaged with respect to the white noise $\mathcal{L}_{u}^{w}$ and the isotropic white noise correlation matrix $\mathcal{D}^{w}$ is given in (21). Below threshold $(\lambda<1)$ the drift matrices are diagonal, yielding diagonal stationary covariance matrices with coefficients (normalized to the shot noise level)

$$
\begin{array}{ll}
\sigma_{x_{a}}^{w}=\frac{1}{(1-\lambda)}, & \sigma_{x_{b}}^{w}=1, \\
\sigma_{y_{a}}^{w}=\frac{1}{(1+\lambda)}, & \sigma_{y_{b}}^{w}=1 .
\end{array}
$$

Quadrature squeezing occurs when the fluctuations fall below 1 , the shot noise level. As found by Milburn and Walls [20] squeezing in the subharmonic increases with $\lambda$ to a maximum $\sigma_{y_{a}}^{w}=1 / 2$ at threshold. The squeezed state is, however, not minimal. The pump mode displays isotropic stationary vacuum fluctuations. Above the threshold for parametric oscillation $(\lambda>1)$, the intermode coupling leads to nondiagonal stationary covariance matrices. The internal stationary normalized variances are given in this case by

$$
\begin{gathered}
\sigma_{x_{a}}^{w}=1+\frac{1}{r}+\frac{1}{2(\lambda-1)}, \quad \sigma_{x_{b}}^{w}=1+\frac{1}{r}, \\
\sigma_{y_{a}}^{w}=\frac{(2 \lambda-1) r^{2}+2 \lambda r}{2 \lambda r^{2}+4 \lambda r}, \quad \sigma_{y_{b}}^{w}=\frac{\lambda r+\lambda+1}{\lambda(2+r)} .
\end{gathered}
$$

It is found that the signal and pump phase components $y_{a}, y_{b}$ display both squeezing for arbitrary pump to subharmonic loss ratio $r$.

We now proceed to investigate how the steady-state properties of the squeezed phase $\mathbf{y}$ components change by the presence of nonwhite pump stochasticity. The phase quadrature correlation matrix $\Sigma_{y}$ is written in terms of the memory integral (25) averaged over the total noise source $\mathcal{L}_{y}$ $=\mathcal{L}_{y}^{w}+\mathcal{L}_{y}^{c}$ as

$$
\begin{aligned}
\Sigma_{y} & =\lim _{\tau \rightarrow \infty} \int_{0}^{\tau} d \tau^{\prime} \int_{0}^{\tau} d \tau^{\prime \prime} e^{\mathcal{A}_{y}\left(\tau-\tau^{\prime}\right)}\left\langle\mathcal{L}_{y}\left(\tau^{\prime}\right) \mathcal{L}_{y}^{T}\left(\tau^{\prime \prime}\right)\right\rangle e^{\mathcal{A}_{y}^{T}\left(\tau-\tau^{\prime \prime}\right)} \\
& =\lim _{\tau \rightarrow \infty} \int_{0}^{\tau} d \tau^{\prime} \int_{0}^{\tau} d \tau^{\prime \prime} e^{\mathcal{A}_{y}\left(\tau-\tau^{\prime}\right)}\left[\mathcal{D}^{w} \delta\left(\tau^{\prime}-\tau^{\prime \prime}\right)+\mathcal{D}_{y}^{c}\left(\left|\tau^{\prime}-\tau^{\prime \prime}\right|\right)\right] e^{\mathcal{A}_{y}^{T}\left(\tau-\tau^{\prime \prime}\right)} .
\end{aligned}
$$

Equation (29) allows us to decompose $\Sigma_{y}$ into independent white and colored noise parts:

$$
\Sigma_{y}=\Sigma_{y}^{w}+\Sigma_{y}^{c}
$$

The first term $\Sigma_{y}^{w}$ represents the $\delta$-correlated contribution of the phase quadrature noise, including the coherent part of the pump and is given by Eq. (26), while the second term $\Sigma_{y}^{c}$ gives the effect of colored pump fluctuations (12) or (14) (representing the incoherent part of the pump field). The total normalized phase quadrature variances averaged over the white and the isotropic colored noise sources are then expressed as

$$
\sigma_{y_{z}}=\sigma_{y_{z}}^{w}+\sigma_{y_{z}}^{c}\left[d, \tau_{c}\right], \quad z=a, b
$$

The case of squeezed colored pump noise yields from (12)

$$
\sigma_{y_{z}}=\sigma_{y_{z}}^{w}-\cos ^{2}(\varphi) \sigma_{y_{z}}^{c}\left[d_{+}, \tau_{+}\right]+\sin ^{2}(\varphi) \sigma_{y_{z}}^{c}\left[d_{-}, \tau_{-}\right]
$$

In order to calculate the contribution of each exponentially correlated noise term of the form $r d \tau_{c}^{-1} e^{-\left|\tau^{\prime}-\tau^{\prime \prime}\right| \tau_{c}^{-1}}$ in $\mathcal{D}_{y}^{c}\left(\left|\tau^{\prime}-\tau^{\prime \prime}\right|\right)$, the exponentiated drift matrix $\mathcal{A}_{y}$ is evaluated, the double memory integral is performed, and the limit $\tau \rightarrow \infty$ is taken. Below threshold the diagonal drift matrices 
(22) decouple the field modes resulting in a nonvanishing contribution of the colored pump noise only on the harmonic mode fluctuations,

$$
\sigma_{y_{a}}^{c}=0, \quad \sigma_{y_{b}}^{c}\left[d, \tau_{c}\right]=\frac{4 d}{r \tau_{c}+1} .
$$

The effect of finite correlation time of the noise is seen in the product $r \tau_{c}$ appearing in (33). The total normalized phase quadrature variance is obtained according to (31) or (32) with $\sigma_{y_{z}}$ given by (27). Above threshold, diagonalization of the exponentiated drift matrices leads to the following colored noise contributions to the stationary normalized subharmonic and harmonic quadrature variances:

$$
\begin{aligned}
& \sigma_{y_{a}}^{c}\left[d, \tau_{c}\right]=\frac{r d}{\tau_{c}} \frac{8 r(\lambda-1)}{\left(\theta_{-}^{y}-\theta_{+}^{y}\right)^{2}}\left[\mathcal{F}_{y_{a}}+\mathcal{G}_{y_{a}}\right], \\
& \mathcal{F}_{y_{a}}=\frac{1}{\left(\theta_{-}^{\left.y^{2}-\tau_{c}^{-2}\right)}\right.}\left\{1+\theta_{-}^{y^{-1}} \tau_{c}^{-1}-\frac{2 \tau_{c}^{-1}}{\left(\theta_{+}^{y}+\theta_{-}^{y}\right)}-\frac{\theta_{-}^{y}-\tau_{c}^{-1}}{\theta_{+}^{y}-\tau_{c}^{-1}}\right\}, \\
& \mathcal{G}_{y_{a}}=\mathcal{F}_{y_{a}}\left[\theta_{+}^{y} \leftrightarrow \theta_{-}^{y}\right], \\
& \sigma_{y_{b}}^{c}\left[d, \tau_{c}\right]=\frac{r d}{\tau_{c}} \frac{4}{\left(\theta_{-}^{y}-\theta_{+}^{y}\right)^{2}}\left[\mathcal{F}_{y_{b}}+\mathcal{G}_{y_{b}}\right],
\end{aligned}
$$

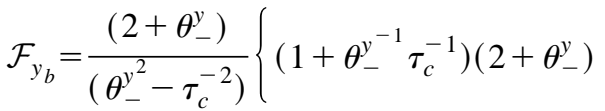

$$
\begin{aligned}
& \left.-\left\{\frac{2 \tau_{c}^{-1}}{\left(\theta_{+}^{y}+\theta_{-}^{y}\right)}+\frac{\theta_{-}^{y}-\tau_{c}^{-1}}{\theta_{+}^{y}-\tau_{c}^{-1}}\right\}\left(2+\theta_{+}^{y}\right)\right\}, \\
& \mathcal{G}_{y_{b}}=\mathcal{F}_{y_{b}}\left[\theta_{+}^{y} \leftrightarrow \theta_{-}^{y}\right]
\end{aligned}
$$

When $(2+r)^{2}-8 r \lambda<0$, the phase eigenvalues $\theta_{ \pm}^{y}$ become complex conjugate of each other, leading to $\mathcal{G}_{y_{z}}=\mathcal{F}_{y_{z}}^{*}$ since $\mathcal{G}_{y_{z}}$ is obtained from $\mathcal{F}_{y_{z}}$ by interchanging the eigenvalues. The total quadrature variances are given formally by (31) or (32) with $\sigma_{y_{z}}^{w}$ now given by Eqs. (28). In the limit of small pump to subharmonic loss ratio $r \rightarrow 0$, the isotropic noise description yields

$$
\begin{gathered}
\lim _{r \rightarrow 0} \sigma_{y_{a}}=\frac{1}{2}=\lim _{r \rightarrow 0} \sigma_{y_{a}}^{w}, \\
\lim _{r \rightarrow 0} \sigma_{y_{b}}=\left[\frac{1}{2}+\frac{1}{2 \lambda}\right]+\frac{4 d}{\lambda},
\end{gathered}
$$

while for the case of squeezed pump noise

$$
\begin{gathered}
\lim _{r \rightarrow 0} \sigma_{y_{a}}=\frac{1}{2}, \\
\lim _{r \rightarrow 0} \sigma_{y_{b}}=\left[\frac{1}{2}+\frac{1}{2 \lambda}\right]+\frac{4}{\lambda}\left[-\cos ^{2}(\varphi) d_{+}+\sin ^{2}(\varphi) d_{-}\right] .
\end{gathered}
$$

From now on, a particular appropriate choice of phase $\varphi=0$ for the squeezed vacuum fed to the pump is made, corresponding to the same relative phase between the coher-
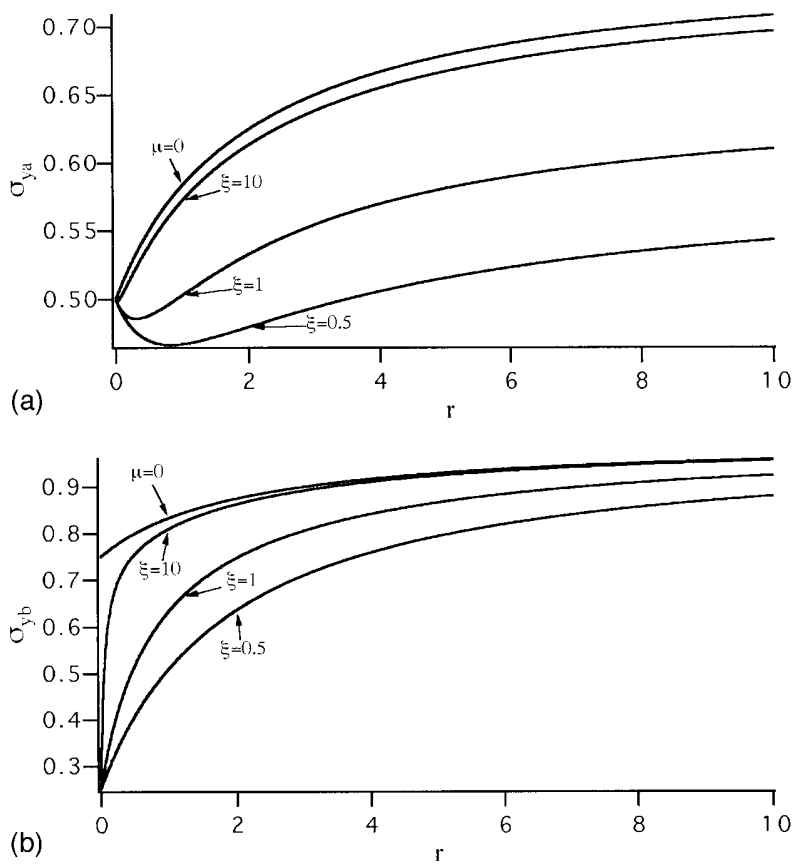

FIG. 1. Normalized intracavity stationary quadrature variance of (a) the subharmonic and (b) the pump field modes for $\lambda=2$ vs the pump to subharmonic loss ratio $r . \mu=0$ refers to the case of coherent pumping and the different $\xi$ values to the correlation times of the stochastic squeezed vacuum noise fed to the pump mode and characterized by a high squeezing strength $\mu=0.9$ [see Eq. (13)].

ent part of the pump (determining the squeezing direction of the oscillator) and the squeezed vacuum field. Such a choice maximizes the steady-state internal phase quadrature squeezing produced by the parametric oscillator with squeezed pump. It is seen that Eqs. (36) and (37) do not depend on the noise correlation time proportional to $\xi$ of the stochastic squeezed pump noise, in particular the same fluctuation levels are found in this limit for broad- and narrow-band stochastic squeezing entering the harmonic mode. In Figs. 1(a) and 1(b), the normalized intracavity subharmonic and pump phase quadrature variances above threshold as given by Eq. (32) are displayed as a function of the pump to subharmonic loss ratio $r$ for $\lambda=2$. The external degenerate parametric amplifier producing the squeezing in the pump is characterized by a driving strength $\mu=0.9$ (large squeezing) and a scaled squeezing bandwidth proportional to $\xi^{-1}$ given by Eq. (13), the limit $\xi \rightarrow 0$ corresponding to the squeezed white noise situation. Two different behaviors are observed concerning the steady-state subharmonic fluctuations: an interplay between $r$ and $\xi$ leads to an initial noise reduction below the value of $1 / 2$ (50\% phase quadrature squeezing) for small $r$ values until a minimum optimum noise level is reached, after which the fluctuations are increased. The position of this minimum moves to the right (bigger $r$ values) and down (lower noise levels) as $\xi \rightarrow 0$. Squeezing is also observed for the pump phase quadrature in the presence of normal coherent pumping. The addition of a squeezed component brings a further noise reduction by an amount equal to $4 d_{+} / \lambda$, according to (37) as $r \rightarrow 0$. The smallest degradation of squeezing is found near the squeezed white noise limit $\xi \rightarrow 0$ as $r$ increases both for the pump and subharmonic 

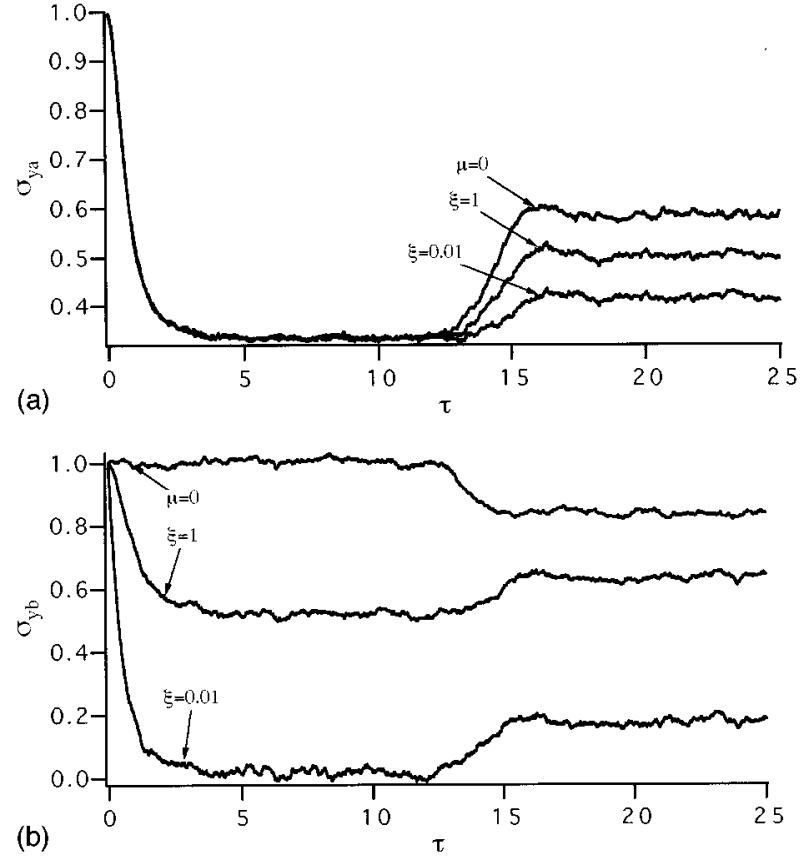

FIG. 2. Time evolution of the normalized intracavity quadrature variance of (a) the subharmonic and (b) the pump field modes for $\lambda=2$ and $r=1 . \mu=0.9$ characterizes the squeezing strength of the stochastic squeezed vacuum injected into the pump mode.

fields. It follows that a region of steady-state squeezing stronger than $50 \%$ exists for the intracavity harmonic and subharmonic phase quadratures of the parametric oscillator when stochastic squeezed vacuum fluctuations are fed to the pump mode. These results are in contrast to those found for the normal case of coherent driving field [20]. The enhancement of squeezing given by Eqs. (34) is, however, in general much less than the squeezing fed to the pump due to pump depletion. This will be clearly seen from the simulations in the next section, comparing the steady-state noise levels of Fig. 2(a) and the transient squeezing level in the pump in Fig. 2(b), for example.

\section{STOCHASTIC SIMULATION OF THE NONLINEAR EQUATIONS}

In previous studies of the degenerate parametric oscillator, the technique of numerical simulation was successfully applied to establish the transient behavior of the system using the positive- $P$ representation [19]. As discussed in the Introduction, we shall adopt here a semiclassical point of view based on a small quantum noise limit allowing for a treatment in the Wigner representation. We shall choose the intracavity pump photon number at threshold $n_{\text {th }}$ $=\gamma_{a} \gamma_{b} / \eta^{2}=r / \widetilde{\eta}^{2}$ equal to $10^{10}$ (i.e., $g^{2}=5 \times 10^{-11}$ ). The stochastic equations (11) are simulated using the traditional Euler algorithm of stochastic integration. The noise sources are constructed by summation of appropriately weighted Gaussian distributed random numbers. To simulate Gaussian exponentially correlated colored noise, the algorithm of Ref. [25] has been adopted. The negative correlations characterizing squeezing require the use of complex random numbers. This enables the quadratures to develop imaginary parts, but these average to zero after a sufficient number of trajectories. All averages have been computed from $2 \times 10^{4}$ trajectories and the integration time step taken was $\Delta \tau=10^{-3}$.

Wolinsky and Carmichael [26] in their treatment of parametric oscillation above threshold neglecting pump depletion suggested that the predicted intracavity field squeezing in a degenerate parametric oscillator operated below threshold, as given by Eq. (27), should be extended above threshold during an initial transient time interval if the system starts in the unstable vacuum steady state while fluctuations in the amplified $x_{a}$ quadrature diverge. This corresponds to the linearized analysis (22) about the unstable steady state (i). When the pump exhibits squeezed stochasticity, the transient squeezing level is obtained according to (32), i.e., replacing $d, \tau_{c}$ in (31) by $-d_{+}, \tau_{+}$, leading to

$$
\sigma_{y_{a}}=\sigma_{y_{a}}^{w}=\frac{1}{1+\lambda}, \quad \sigma_{y_{b}}=1-\frac{\mu}{\lambda_{+}\left(\lambda_{+}+\xi r\right)}
$$

in terms of the squeezing parameters defined in (13). It is thus apparent that the incoherent stochastic component of the pump field does not alter the transient intracavity subharmonic squeezing, which depends only on the driving power $\lambda$. Perfect transient squeezing is still achieved in the limit $\lambda \rightarrow \infty$ without pump depletion [26] for the system beginning in the unstable state. The level of transient noise in the harmonic field is fixed from (33) by the product of the noise correlation time $\tau_{c}$ and the pump to subharmonic loss ratio $r$ (for fixed noise intensity), i.e., for the squeezed pump case, by the product $\xi r$ as seen in (38).

Our aim is to confirm these basic results by numerical simulation of the nonlinear equations (11) and to analyze the effect of stochastic pump noise on the turn on of pump depletion, i.e., once the system senses the nonlinearity of the mode coupling causing bistability. The oscillator has been chosen to operate at driving strength $\lambda=2$ and when dealing with squeezed pump, the value $\mu=0.9$ will characterize the squeezing strength in all simulations. The initial state of the cavity corresponds to the unstable vacuum state for both modes. Figure 2 provides a comparison between the time evolution of the squeezing attained with normal coherent pump and squeezed pump for equal pump and subharmonic decay rates $(r=1)$. The initial decay of the fluctuations governed by the eigenvales $\theta_{ \pm}^{y}$ reaches a transient constant minimum squeezing level given by Eqs. (38),

$$
\sigma_{y_{a}}=1 / 3, \quad \sigma_{y_{b}} \sim 1-1 /(1+\xi r)
$$

before the unstable state starts decaying. Once the nonlinearity associated to pump depletion becomes important, the system evolves to one of the two possible steady states, resulting in the degradation of the transient squeezing. The use of a squeezed pump reduces this degradation depending on the squeezing bandwidth proportional to $\xi^{-1}$ of the noise injected into the pump. On the other hand, for the pump mode, the effect of pump depletion is to reduce fluctuations below the shot noise level in the case of coherent pumping but to enhance in general the intracavity squeezed fluctuations associated with the squeezed vacuum entering the pump cavity mode in the case of squeezed pumping. Both for the subharmonic and harmonic fields, maximum steady-state intracav- 


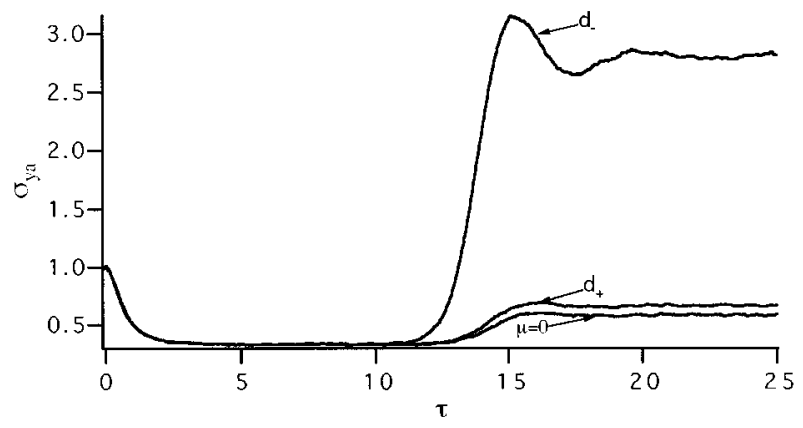

FIG. 3. Time evolution of the normalized intracavity subharmonic quadrature variance for $\lambda=2$ and $r=1$ when isotropic noise is fed to the coherent pump field. $\mu=0$ denotes the coherent pump case. The isotropic noise parameters $d, \tau_{c}$ are equal to the squeezed noise parameters $d_{ \pm}, \tau_{ \pm}$given by Eq. (13) with $\mu=0.9$ and $\xi=1$, i.e., $d_{+} \sim 1 / 4, \tau_{+} \sim 1, d_{-}=90, \tau_{-}=20$.

ity squeezing is obtained near the white noise limit $\xi \rightarrow 0$ as shown for $\xi=1$ and $10^{-2}$. We note that the linearized stationary fluctuations given by Eqs. (28) and (34) are in agreement with the steady-state values obtained from the numerical simulation of Eqs. (11), demonstrating thereby the validity of our semiclassical treatment of the system dynamics. The case of isotropic stochastic noise fed to the pump as given by (14) is investigated in Fig. 3. We consider the noise parameters of the previous figure, $\mu=0.9, \xi=1$ giving $d_{+} \sim 1 / 4, d_{-}=90$ and $\tau_{+} \sim 1, \tau_{-}=20$. The isotropic noise characterized by the parameters $d, \tau_{c}$ is compared to the squeezed noise description by equaling $d$ to $d_{ \pm}$and $\tau_{c}$ to $\tau_{ \pm}$; i.e., the noises are compared at equal intensity and correlation time. We see that the duration of the transient
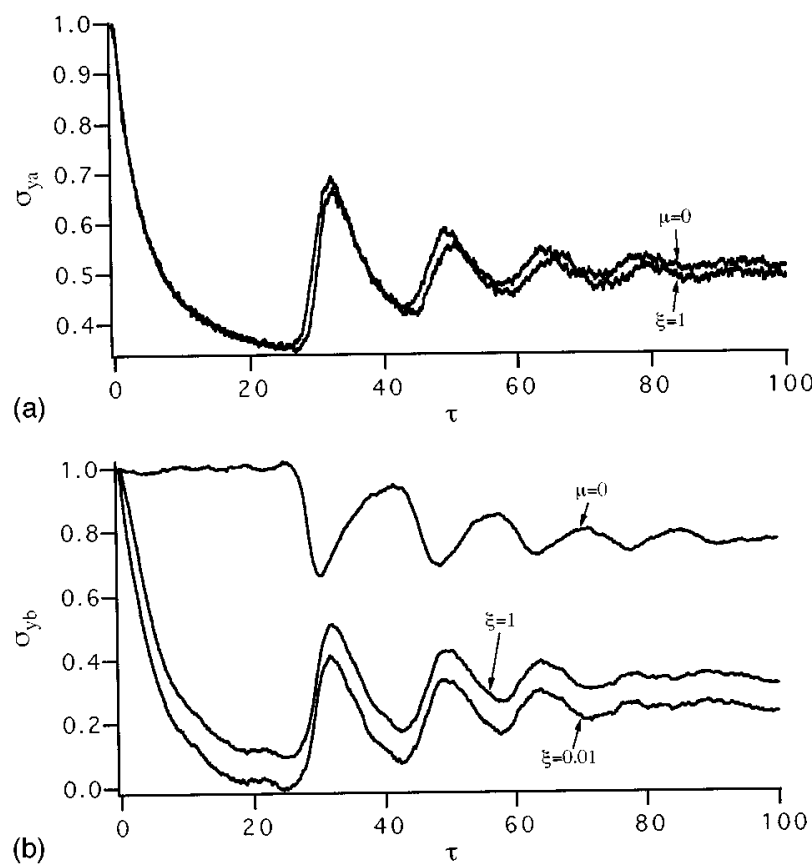

FIG. 4. Time evolution of the normalized intracavity quadrature variance of (a) the subharmonic and (b) the pump field modes for $\lambda=2$ and $r=0.1 . \mu=0.9$ characterizes the squeezing strength of the stochastic squeezed vacuum injected into the pump mode.
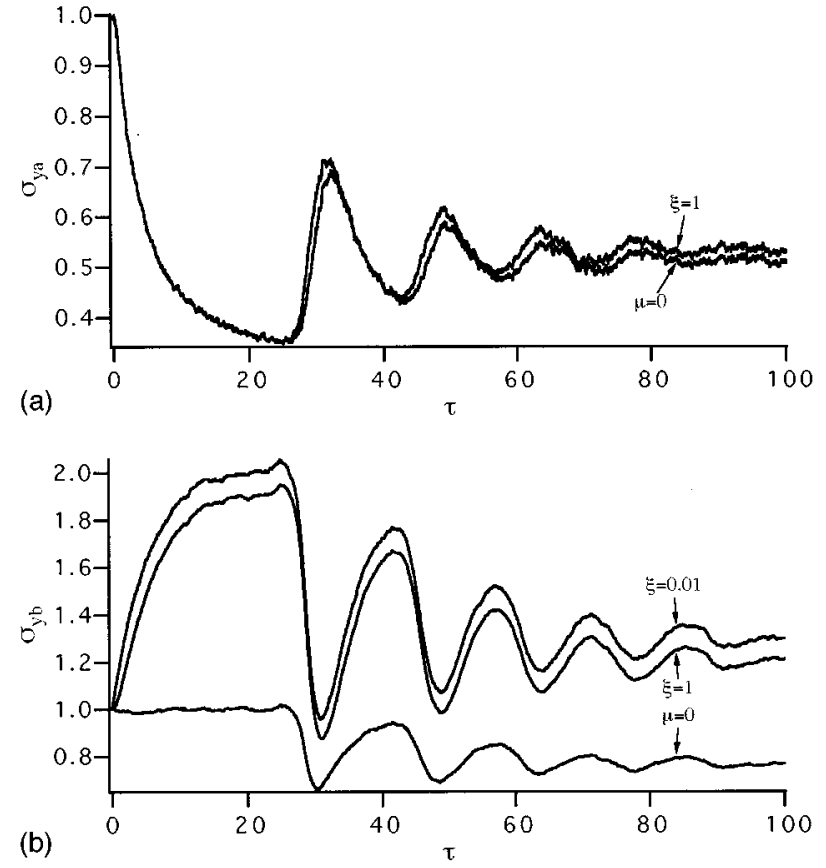

FIG. 5. Time evolution of the normalized intracavity subharmonic quadrature variance for $\lambda=2$ and $r=0.1$ when isotropic noise is fed to the coherent pump field. $\mu=0$ denotes the coherent pump case. The isotropic noise parameters $d, \tau_{c}$ are equal to the squeezed noise parameters $d=d_{+}, \tau_{c}=\tau_{+}$given by Eq. (13) with $\mu=0.9$ and $\xi=1,10^{-3}$, i.e., $d \sim 1 / 4, \tau_{c} \sim 1,10^{-3}$.

squeezing is nearly not affected by the presence of isotropic pump noise. Only when $d, \tau_{c}$ are equal to the noise parameters $d_{-}, \tau_{-}$do we observe a slightly faster decay of the unstable state bringing the subharmonic amplitude to a steady-state value. This feature is present only in the case of large isotropic pump fluctuations varying on a very slow time scale (as the unsqueezed amplitude quadrature fluctuations of the pump in the case of strong squeezed pumping). The steady-state subharmonic noise level is given by Eqs. (28) and (33). We note the different sign of the colored noise term compared to the case of squeezed pump, leading to excess noise. In Fig. 4 are plotted simulations for small pump to subharmonic loss ratio, $r=0.1$ with coherent and squeezed pumping. The slow time scale associated to the

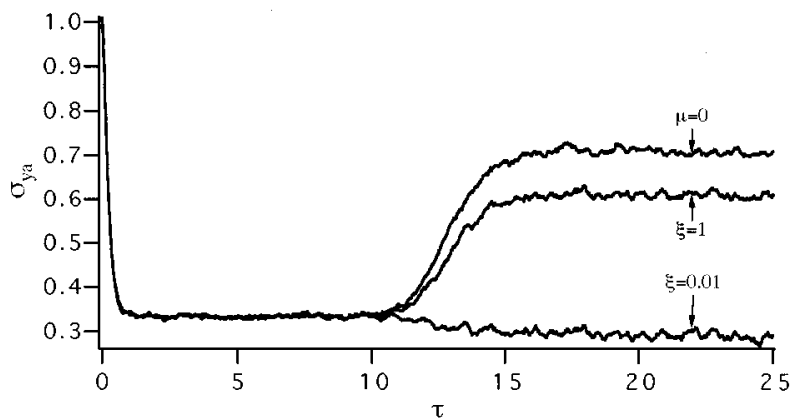

FIG. 6. Time evolution of the normalized intracavity subharmonic quadrature variance for $\lambda=2$ and $r=10 . \mu=0.9$ characterizes the squeezing strength of the stochastic squeezed vacuum injected into the pump mode. 
overall dynamical evolution of the fluctuations (governed essentially by the eigenvalues of the associated drift matrix) as a consequence of the small $r$ value chosen does not display a constant transient squeezing level. When the unstable state starts decaying due to the nonlinearity associated to the depletion of the pump mode, an interesting damped oscillatory behavior of the fluctuations acompanying the growth in amplitude of the subharmonic field is observed (periodic exchange of noise between the phase quadrature of the cavity modes). The minimum subharmonic and harmonic transient squeezing levels are given by Eqs. (39). A comparison with the case of isotropic stochastic pump characterized by noise parameters $d=d_{+}, \tau_{c}=\tau_{+}$is presented in Fig. 5. The same global evolution pattern as in the previous figure is found, the incoherent isotropic pump noise exhibiting excess noise in contrast to the reduced fluctuations of the previous case. The maximum noise level in the pump field before the decay of the fluctuations due to pump depletion is given by with

$$
\sigma_{y_{b}} \sim 1+1 /(1+\xi r) .
$$

Finally, the overdamped regime ( $r \gg 1)$ is displayed in Fig. 6 for $r=10$ and other parameters as given in Fig. 2. The use of a broadband squeezed pump brings in this case the steadystate fluctuations below the constant transient level.

\section{CONCLUSION}

This paper has investigated the effects of stochastic colored pump noise on the dynamical intracavity squeezing produced in parametric oscillators operated above threshold. A semiclassical treatment in the Wigner representation founded on the grounds of a very small quantum noise limit has been used. Comparison between stochastic colored squeezed and isotropic pump noises has shown that the transient squeezing predicted by Wolinsky and Carmichael [26] for the system starting in the unstable state is not degraded by the stochasticity of the driving field. In the limit of large signal cavity mode decay $(r \ll 1)$, damped oscillations of the fluctuations have been found on the turn on of the nonlinearity associated to the depletion of the pump mode. Finally, we have also shown that the interplay between the correlation time of the noise injected into the driving field and the pump to subharmonic loss ratio $r$ allows the steady-state subharmonic and harmonic noise levels to lie below the limit value of $50 \%$ squeezing found in the subharmonic field in the case of normal coherent pumping.

\section{ACKNOWLEDGMENTS}

L. G. wishes to acknowledge J. Martínez-Linares for helpful suggestions on the subject, and C. Cabrillo and E. Hernández-García for their advice on the numerical simulations. This work has been supported by the European Union under Contract No. ERB4050PL931060.
[1] L.A. Wu, H.J. Kimble, J.L. Hall, and H. Wu, Phys. Rev. Lett. 57, 2520 (1986); P. Grangier, R.E. Slusher, B. Yurke, and A. La Porta, ibid. 59, 2153 (1987).

[2] N. Bloembergen, Nonlinear Optics (Benjamin, New York, 1965).

[3] J.A. Armstrong, N. Bloembergen, J. Ducuing, and P.S. Pershan, Phys. Rev. 127, 1918 (1962).

[4] R. Graham and H. Haken, Z. Phys. 210, 276 (1968); R. Graham, ibid. 210, 319 (1968); 211, 469 (1968).

[5] R. Graham, in Quantum Statistics in Optics and Solid-State Physics, edited by G. Hohler, Springer Tracts in Modern Physics Vol. 66 (Springer, New York, 1973), p. 1.

[6] P.D. Drummond, K.J. McNeil, and D.F. Walls, Opt. Acta 27, 321 (1980).

[7] P.D. Drummond and C.W. Gardiner, J. Phys. A 13, 2353 (1980).

[8] H.J. Carmichael, An Open Systems Approach to Quantum Optics (Springer-Verlag, Berlin, 1993).

[9] B. Yurke, P.G. Kaminsky, R.E. Miller, E.A. Whittaker, A.D. Smith, A.H. Silver, and R.W. Simon, Phys. Rev. Lett. 60, 764 (1988)

[10] L. Krippner, W.J. Munro, and M.D. Reid, Phys. Rev. A 50, 4330 (1994).

[11] P.D. Drummond, K.J. McNeil, and D.F. Walls, Opt. Acta 28, 211 (1981).

[12] M. Wolinsky and H.J. Carmichael, Phys. Rev. Lett. 60, 1836 (1988).

[13] D.L. Mills, Nonlinear Optics (Springer-Verlag, Berlin, 1991).
[14] C.W. Gardiner, Quantum Noise (Springer, Berlin, (1991).

[15] R. Bonifacio and L.A. Lugiato, Phys. Rev. A 18, 1129 (1978); T.S. Agarwal, L.M. Narducci, D.H. Feng, and F. Gilmore, ibid. 18, 620 (1978).

[16] P. Kinsler and P.D. Drummond, Phys. Rev. Lett. 64, 236 (1989); P.D. Drummond and P. Kinsler, Phys. Rev. A 40, 4813 (1989).

[17] W.J. Munro and M.D. Reid, Quantum Opt. 4, 181 (1992).

[18] E.P. Wigner, Phys. Rev. 40, 749 (1932).

[19] H.J. Carmichael and M. Wolinsky, Quantum Optics 4, Springer Proceedings in Physics Vol. 12 (Springer, Berlin, 1986), p. 208.

[20] G. Milburn and D.F. Walls, Opt. Commun. 39, 401 (1981).

[21] W.H. Louisell, Quantum Statistical Properties of Radiation (Wiley, New York, 1973).

[22] H.J. Kimble, Fundamental Systems in Quantum Optics, edited by J. Dalivard, J.M. Raimond, and J. Zinn-Justin (NorthHolland, Amsterdam, 1992), p. 545.

[23] P. Kinsler and P.D. Drummond, Phys. Rev. A 43, 6194 (1991); 44, 7848 (1991).

[24] C.W. Gardiner, Handbook of Stochastic Methods (Springer, Berlin, 1985); H. Risken, The Fokker-Planck Equation (Springer, Berlin, 1989).

[25] E. Hernández-García, R.Toral, and M. San Miguel, Phys. Rev. A 42, 6823 (1990).

[26] M. Wolinsky and H.J. Carmichael, Opt. Commun. 55, 138 (1985). 